\title{
Ballon aortic valvuloplasty: an obsolete method in intervention cardiology that still extends life
}

\section{Marina Pavletić, Siniša Blažon, Ivana Barun}

University Hospital Centre Zagreb, Zagreb, Croatia

\author{
KEYWORDS: ballon aortic valvuloplasty, aortic vale. \\ CITATION: Cardiol Croat. 2018;13(1-2):59. I https://doi.org/10.15836/ccar2018.59
}

*ADDRESS FOR CORRESPONDENCE: Marina Pavletić, Klinički bolnički centar Zagreb, Kišpatićeva 12, HR-10000 Zagreb, Croatia. / Phone: +385-95-562-6804 / E-mail: marinapavletic@gmail.com

ORCID:Marina Pavletić, https://orcid.org/0000-0002-1165-7097 • Siniša Blažon, https://orcid.org/0000-0002-4799-2567

|IIIIIIIIIIIIIIIIIIIIIIIIIIIIIIIIIIIIIIIIIIIIIIIIIIIIIIIIIIIIIIIIIIIIIIIIIIIIIIIIIIIIIIIIIIIIIIIIIIIIIIIIIIIIIIIIIIIIIII

Ballon aortic valvuloplasty (BAV) was introduced more than three decades as an alternative to replacement of aortic valve in older patients who are considered unsuitable for surgery. ${ }^{1}$ Because of the high rate of early restenosis and without affecting long-term survival, the development of the procedure has stopped. However, with recent technological advances, especially with the appearance of a TAVI, there was a revival of this technique. Balloon aortic valvuloplasty is a percutaneous therapeutic option for patients with severe aortic stenosis but the effectiveness of this procedure depends on the morphology of the stenotic aortic valve and the respective dilation mechanism. In younger patients with congenital aortic stenosis, there are good acute and mid-term results.

In this paper, we describe and present the preoperative course, the performance of balloon aortic valvuloplasty and postprocedural process. BAV allows patients with severe aortic stenosis to safely survive to surgery, to TAVI or to relieve symptoms by conservative therapy.
RECEIVED:

February 4, 2018

ACCEPTED:

February 10, 2018

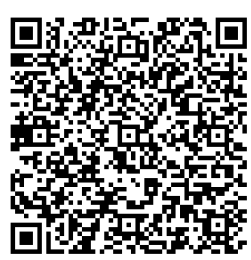

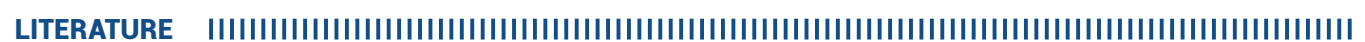
1. Kern MJ. The Interventional Cardiac Catheterization 2th ed. St. Louis: Mosby; 2004. 